\title{
Autism spectrum disorder, anxiety and severe depression in a male patient with deletion and duplication in the 21q22.3 region: A case report
}

\author{
SANDRO ORRU $^{1}$, IOANNIS PAPOULIDIS ${ }^{2}$, ELISAVET SIOMOU ${ }^{2}$, DIMITRIOS T. PAPADIMITRIOU ${ }^{3,4}$, \\ SOTIRIOS SOTIRIOU ${ }^{5}$, PETROS NIKOLAIDIS ${ }^{6}$, MAKARIOS ELEFTHERIADES ${ }^{7}$, \\ EVAGGELOS PAPANIKOLAOU $^{8}$, LORETTA THOMAIDIS $^{9}$ and EMMANOUIL MANOLAKOS ${ }^{1,2,9}$
}

${ }^{1}$ Department of Medical Genetics, University of Cagliari, Binaghi Hospital, I-09126 Cagliari, Italy; ${ }^{2}$ ATG P.C., Clinical Laboratory Genetics, Athens $11528 ;{ }^{3}$ Department of Pediatric Endocrinology and Diabetes, Athens Medical Center, Athens 15125; ${ }^{4}$ Pediatric Endocrine Unit, Attikon University Hospital, Athens 12462;

${ }^{5}$ Department of Clinical Embryology, Larissa Medical School, University of Thessaly, Larissa 41334;

${ }^{6}$ Embryoiatriki-genetiki Ltd., Athens $15125 ;{ }^{7}$ Second Department of Obstetrics and Gynecology,

Aretaieio Hospital, Medical School, National and Kapodistrian University of Athens, Athens 11528;

${ }^{8}$ Third Department in Obstetrics and Gynecology, Ippokratio Hospital, Aristotle University of Thessaloniki,

Thessaloniki 54642; ${ }^{9}$ Developmental Assessment Unit, Second Department of Pediatrics, 'P. \& A. Kyriakou'

Children's Hospital, School of Medicine, National and Kapodistrian University of Athens, Athens 11527, Greece

Received July 27, 2018; Accepted March 27, 2019

DOI: $10.3892 /$ br.2019.1210

\begin{abstract}
In this report, a patient carrying a $650 \mathrm{~kb}$ deletion and a $759 \mathrm{~kb}$ duplication of chromosomal 21q22.3 region was described. Facial dysmorphic features, hypotonia, short stature, learning impairment, autism spectrum disorder, anxiety and depression were observed clinical characteristics. Mentioned copy number variants were the shortest in length reported so far. The current study hypothesized that the presence of a susceptibility locus for autism spectrum disorder associated with depression and anxiety may be located in a $200 \mathrm{~kb}$ region between the PCNT and PRMT2 genes. The current study aimed to provide insight into the human genome morbidity map of chromosome 21 .
\end{abstract}

\section{Introduction}

Chromosomal imbalances are frequently associated with dysmorphism, congenital malformations and psychomotor impairment (1). Aneuploidies involving human chromosome 21 (Hsa21) are the most prevalent chromosomal abnormalities in humans, with down syndrome (DS) describing the presence of an extra copy of Hsa21, occurring in $\sim 1$ of 733 live births (2), and monosomy 21 being very rare $(<0.01 \%)$ resulting in

Correspondence to: Dr Emmanouil Manolakos, ATG P.C., Clinical Laboratory Genetics, 8 Sisini Street, Athens 11528, Greece E-mail:manolakos@atg-labs.gr

Key words: autism spectrum disorder, anxiety, depression, 21q22.3 mortality in utero or shortly following birth (3). Partial monosomy and trisomy are difficult to detect with conventional cytogenetic techniques (1). Novel technologies, including high-resolution microarray comparative genomic hybridization $(\mathrm{aCGH})$, quantitative polymerase chain reaction (qPCR) and fluorescent in situ hybridization (FISH) allow identifying chromosomal imbalances and aid correlating geno- and phenotypes in patients (1). This information may be used in genetic counseling, which is an aim of the present study.

Partial deletion of Hsa21 is more frequent than complete monosomy and patients have increased survival expectancy and are heterogeneous regarding their phenotypic severity depending on the position and size of the deleted region (4-7). There are $>45$ cases reported in literature. Lyle et al (6) differentiate three regions associated with various levels of clinical severity, ranging from moderate to not compatible with life. The deleted region described in this study was consistent with that Roberson et al (7) reported as the most frequently altered and with the mildest phenotypic expression.

The occurrence of segmental trisomy 21, with or without association to DS, was first reported by Ilbery et al (8) in 1961. Approximately 200 cases have been reported in literature until 2016 compared with an estimated 5.8 million patients with DS worldwide (9). It has been demonstrated that only part of the Hsa21 is involved in the pathogenesis of DS (9). Pelleri et al (9) reported a highly restricted DS critical region of $34 \mathrm{~kb}$, with no known genes located in it and it was identified as the minimal region duplicated patients with DS and absent healthy subjects.

The present study described a patient carrying a 21q22.3 deletion and duplication as detected by aCGH array. Symptoms included facial dysmorphism, hypotonia, short stature, clinodactyly, learning impairment, autism spectrum 
disorder (ASD), anxiety and depression. To the best of our knowledge this is the first case reporting these two copy number variants (CNVs) to occur simultaneously and these regions were the shortest described so far. The analysis of these partial aneuploidies aimed to correlate clinical features of the phenotype to this specific Hsa21 region.

\section{Clinical report}

Patient details. A full-term male born to unrelated, healthy parents following an uncomplicated pregnancy was reported. The patient first presented at the Developmental Assessment Unit of the 2nd Department of Pediatrics, 'P. \& A. Kyriakou' Children's Hospital (Athens, Greece) in October 2015. The patient was 10 years at the time of recruitment and he was the first child of the family. The patient was delivered by cesarean section with 3,150 $\mathrm{g}$ birth weight (25th percentile), $50 \mathrm{~cm}$ length (50th percentile) and $34 \mathrm{~cm}$ head circumference (HC; 25th percentile). No abnormalities or complications were recorded in the perinatal history. Developmental milestones were reported normal; the patient sat unsupported at 7 months, walked unaided at 14 months and started speaking at 13 months.

At 10 years old, the patient was referred for full developmental assessment due to learning difficulties and behavioral problems at school, affecting social relationships with peers. Between 10-12 years, the patient received psychological treatment. The patient exhibited a shy demeanor, short statue, microcephaly and minor dysmorphic facial and body features, including long philtrum, frontal bossing, almond shaped eyes, auricle abnormalities, nipples widely spaced and clinodactyly of the fifth finger. At the time of recruitment, the following details were recorded: Height, $130 \mathrm{~cm}$ (3rd percentile); weight, $33 \mathrm{~kg}$ (25th percentile); and $\mathrm{HC}, 51 \mathrm{~cm}$ (<3rd percentile). The intellectual abilities were tested using the Wechsler intelligence scale for children (WISC III) (10) and a difference between verbal and performance skills was determined. The verbal score was at upper limit (verbal $\mathrm{IQ}=111$ ) and the performance score was at lower end (performance $\mathrm{IQ}=83$ ) of the mean IQ range (IQ, 80-119) (10). Child psychiatric evaluation exhibited severe anxiety and depression levels.

At 12 years, a psychiatric evaluation diagnosed autistic spectrum disorder (ASD) according to diagnostic and statistical manual of mental disorders (DSM)-5 criteria (11). Combined educational and pharmaceutical treatment followed (risperidone, $0.25 \mathrm{mg}$ twice daily). Clinical neurological examination revealed global hypotonia without focal neurological signs and microcephaly. Laboratory investigations, including audiological, visual, biochemical, metabolic, endocrine (thyroid, growth hormone, luteinizing hormone, follicle stimulating hormone, adrenocorticotropic hormone and prolactin), bone age, kidney/liver ultra sound, triplex ultrasound, brain magnetic resonance imaging and electroencephalogram were normal.

Materials and methods. Metaphase chromosomes were obtained from phytohemagglutinin-stimulated peripheral blood lymphocytes and high-resolution (thymidine treatment) G-banding karyotype analysis was performed using standard procedures (12).

aCGH was performed by normalizing the sample against a male human reference commercial DNA sample using an
aCGH platform that includes 60,000 oligonucleotides as described elsewhere (9), distributed across the entire genome (cat. no. 5190-3796; Agilent Technologies, Inc., Santa Clara, CA, USA). Statistical analyses estimating the number of copies were performed using Cytogenomics v4.0 (Agilent Technologies, Inc.) with a window of $0.5 \mathrm{Mb}$ and $\mathrm{A}=6$. If copy number changes affected $\geq 5$ consecutive probes with identically oriented change, these were considered as CNVs. For the majority of the genome, the mean genomic power of resolution was $200 \mathrm{~kb}$. The genomic coordinates were listed according to genomic build GRCh37/hg19 (genome.ucsc.edu).

\section{Results}

The cytogenetic analysis revealed a normal male karyotype (46,XY). aCGH analysis detected the loss of $\sim 650 \mathrm{~kb}$ and a copy number gain of $\sim 760 \mathrm{~kb}$ in the $21 \mathrm{q} 22.3$ region (Fig. 1). These alterations affected 13 and 11 genes, respectively. The deleted segment was mapped at the chr21:47,439,416-48,090,31 7 region and the duplicated segment was mapped at the chr21:4 2,710,862-43,473,699 region. The deletion spanned ten OMIM genes and was one of the shortest described in the literature so far (Table I). The duplicated region described in the present study covers the proximal $\sim 760 \mathrm{~kb}$ of the $21 \mathrm{q} 22.3$ region and includes eight online Mendelian inheritance in man (OMIM) genes (Table II).

\section{Discussion}

The study of chromosomal abnormalities is the most commonly used tool for the definition of the morbidity map of the human genome (1). In this respect, it is of fundamental importance to study small deletions/duplications $(<1 \mathrm{Mb})$, which may be characterized with high accuracy due to the introduction of aCGH (13).

The current study presented the case of a 12-year old male patient with normal perinatal and infant development. By 10 years, the patient exhibited learning difficulties and behavioral problems, resulting in a referral for full developmental assessment that revealed short stature, microcephaly, hypotonia and minor facial and trunk dysmorphic features. At 10 years, a psychiatric evaluation suggested severe anxiety and depression that strongly conditioned social relations. On an intellectual level, there was a discrepancy between verbal and performance skills. At 12 years, a psychiatric evaluation diagnosed ASD according to DSM-V criteria. Using aCGH, a duplication of $\sim 760 \mathrm{~kb}$ and a deletion of $\sim 650 \mathrm{~kb}$ in the $21 \mathrm{q} 22.3$ region were identified.

Although DS is the most common chromosomal abnormality, 125 cases describing segmental trisomy of Hsa21 have been published in the literature so far (9). Few studies identified the chromosomal region of interest as 21q22 $(6,9)$. Over forty cases of partial trisomy of Hsa21 that include the 21q22.3 region have been described in the literature to date (4-7). A comparison between the present study and the literature has proven difficult due to variations in the size of the duplication and the presence of other chromosomal aberrations. Additionally, certain clinical characteristics described in the present study, including short stature, microcephaly, hypotonia and clinodactyly of fifth finger are associated with DS, but are 


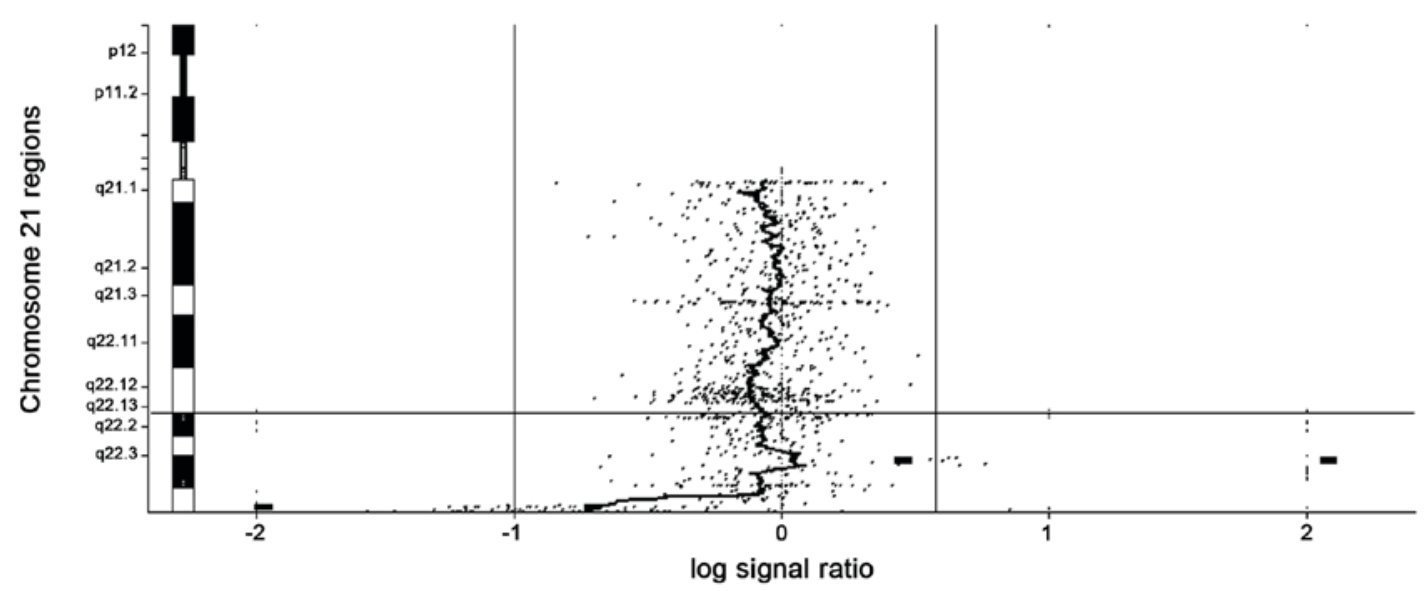

Figure 1. Molecular karyotype of chromosome 21 recorded for the 10 -year-old male patient. A high-resolution microarray comparative genomic hybridization analysis was performed. The physiological value of the signal ratio is 0 , with variations describing abnormal genotypes (signal ratio +0.5 , duplication; signal ratio -1, deletion).

Table I. Deleted OMIM genes determined for the 10-year-old male patient.

\begin{tabular}{lc}
\hline Gene & OMIM reference \\
\hline COL26A2 & 120240 \\
FTCD & 606806 \\
C21orf56 & 612412 \\
LSS & 600909 \\
$M C M 3 A P$ & 603294 \\
YBEY & 617461 \\
PCNT & 605925 \\
DIP2A & 607711 \\
S1OOB & 176990 \\
HRMT1L & 601961 \\
\hline
\end{tabular}

Determined using genome browser, hg19. OMIM, online Mendelian inheritance in man.

also common to other chromosomal abnormalities. Therefore, it was not possible to attribute the characteristics specifically to the partial trisomy 21 .

There is growing evidence that trisomy of the 21q22.3 segment exhibits no clinical significance (14). A terminal duplication of $\sim 5 \mathrm{Mb}$ of the 21q22.3 region was observed in a 2 -year-old male exhibiting no pathological phenotype (15). An interstitial duplication of $4.4 \mathrm{Mb}$ of the 21q22-22.3 region was detected in a 2-year-old healthy female and her 37-year-old healthy mother (16). In a large subtelomere FISH analysis, two cases presented with a $21 \mathrm{q}$ deletion and exhibited no clinical signs of disease (17). Therefore, the pathological characteristics observed in the patient of the present study were suggested to be attributed to a combination of the partial trisomy and the terminal deletion of the distal region of 21q22.3. Comparable combinations of an inverted duplication adjacent to a terminal deletion have been described for chromosome arms 2q, 4p, 5p, $6 q, 8 p, 9 p, 10 q, 13 q, 15 q, 18 p, 18 q$ and 22q (18). The mechanisms proposed for the complex rearrangement are based on non-allelic homologous recombination, U-type exchange and
Table II. Duplicated OMIM genes determined for the 10-year-old male patient.

\begin{tabular}{lc}
\hline Gene & OMIM reference \\
\hline FAM3B & 608617 \\
$M X 2$ & 147890 \\
$M X 1$ & 147150 \\
TMPRSS2 & 602060 \\
RIPK4 & 605706 \\
PRDM15 & 617692 \\
C2CD2 & 617581 \\
ZBTB21 & 616485 \\
\hline
\end{tabular}

Determined using genome browser, hg19. OMIM, online Mendelian inheritance in man.

telomere capture $(18,19)$. For the present case, the underlying mechanisms were not identified and it remains to be investigated whether the duplication is inverted. Future studies may include the use of FISH aiding to identify a potential inversion, and sequencing of the regions in proximity to the breakpoints may reveal the mechanisms of rearrangement.

Lyle et al (6) proposed the classification of partial 21q monosomy into three groups: i) Deletions starting from the centromere up to $32.3 \mathrm{Mb}$; ii) deletions in the $32.2-37.1 \mathrm{Mb}$ region; and iii) deletions from $37 \mathrm{Mb}$ to the telomere. The first two groups of 21q monosomy are associated with clinically severe phenotypes, while the third group exhibits moderate phenotypes (6). Clinical characteristics observed in the present patient frequently reported in the literature for partial Hsa21q monosomy included microcephaly and short stature with growth delay.

A chromosome 21 ring carrying a deletion that includes the genes COL6A2 and $S 100 B$ is propagated in a family. Four individuals from three generations with monosomy were described and patients were diagnosed with a medium to severe growth retardation, short stature and microcephaly (20). McGinniss et al (21) described 13 patients with Hsa21 rings; 
five were clinically healthy and had a deletion with two breakpoints between COL6A1 and $S 100 B$. Mental retardation of mild grade, growth retardation, short stature and microcephaly were observed in a patient with a longer deletion including the COL6Al gene. Roberson et al (7) presented ten novel cases of partial 21q monosomy, including a 4-year-old male with a de novo $5.68 \mathrm{Mb} 21 \mathrm{q} 22.3$ terminal deletion presenting moderate mental retardation, strabismus, normal growth and an absence of dysmorphism. The Hsa21 monosomal region observed in the present case included 10 OMIM genes and to date no genes with pathological phenotype have been mapped in this region.

The patient of the present study exhibited anxiety and severe depression that affected academic performance and relationships with peers at 10 years. The difference between verbal and performance skills evaluated using the WISC-III test was attributed to these symptoms. At 12 years, a psychiatric evaluation diagnosed ASD according to DSM-V criteria. Other family members were not diagnosed with mood disorders. Anxiety and depression are clinical signs rarely reported in patients with chromosomal abnormalities. This may be due to geneticists consulting on pediatric cases and follow-up to when psychiatric disorders manifest are rare. Psychiatrists often do not consider genetic syndromes in the diagnosis of their patients (22). However, depression is difficult to diagnose during childhood as it may be masked by major phenotypes, including even mild mental retardation (23). Therefore, anxiety and depression may be underestimated with respect to actual prevalence. A recent study has suggested that occurrence of depression and anxiety is frequent (20\%) in individuals with ASD and is associated with higher IQs and fewer ASD symptoms (24). Two associated studies have highlighted a susceptibility locus for bipolar depression, which maps to the 21q22 region $(25,26)$. Major depression was observed in 16- and 18-year-old brothers with a terminal deletion of $\sim 200 \mathrm{~kb}$ in the $21 \mathrm{q} 22.3$ region. Further analysis of the deletion revealed monosomy of the PCNT, DIP2A, SIOOB and PRMT2 genes (27). Of interest, certain SNPs of PCNT located in this $200 \mathrm{~kb}$ region have been associated with schizophrenia (28) and major depressive disorder (29). The second candidate gene in the etiology of major mood disorders is $S 100 B$, encoding S100 calcium-binding protein $\mathrm{B}$, which has been associated with the etiology of schizophrenia (30), bipolar depression $(31,32)$ and autism (33). However, these studies predominantly refer to protein overexpression compared with levels observed for healthy controls. In the current case, a downregulation of S100B would be expected due to the haploinsufficiency of the examined region; however, further experiments are required to support this hypothesis. Furthermore, CNVs in the 21q22.3 region, including $D I P 2 A$ and $S 100 B$, have been associated with ASD (34). A frame shift mutation and a splice site variant of DIP2A were observed in two families with an ASD affected member each $(35,36)$. Furthermore, DIP $2 A$ has been associated with the onset of dyslexia (37), a clinical characteristic missing in the patient presented in the current study, but described for patients with monosomy of the $21 \mathrm{q} 22.3$ region (37). DIP2A was suggested to be a fragile $\mathrm{X}$ mental retardation 1 translation regulator target in nervous cells (38).

In conclusion, the present study described the case of a 10 -year old male, where aCGH analysis identified one of the shortest deletion/duplication of the $21 \mathrm{q} 22.3$ region reported to date. This information may guide genetic counseling in the future. Furthermore, it was hypothesized that the presence of a susceptibility locus for ASD may be associated with depression and anxiety; this locus was mapped in a $200 \mathrm{~kb}$ region between PCNT and PRMT2.

\section{Acknowledgements}

Not applicable.

\section{Funding}

No funding was received.

\section{Availability of data and materials}

The datasets used and/or analyzed during the current study are available from the corresponding author on reasonable request.

\section{Authors' contribution}

SO, IP, LT, EM and EP conceived and designed the study. ES, DTP, SS, PN, ME acquired and analyzed the data. All authors read and approved the final version of the manuscript.

\section{Ethics approval and consent to participate}

Written informed written consent was obtained from the patients' parents and the study protocol was approved by the Ethics Committee of the 'P. \& A. Kyriakou' Children's Hospital (Athens, Greece).

\section{Patient consent for publication}

The family of the patient gave a written consent for the publication.

\section{Competing interests}

The authors declare that they have no competing interests.

\section{References}

1. Theisen A and Shaffer LG: Disorders caused by chromosome abnormalities. Appl Clin Genet 3: 159-174, 2010.

2. Canfield MA, Honein MA, Yuskiv N, Xing J, Mai CT, Collins JS, Devine O, Petrini J, Ramadhani TA, Hobbs CA, et al: National estimates and race/ethnic-specific variation of selected birth defects in the United States, 1999-2001. Birth Defects Res A Clin Mol Teratol 76: 747-756, 2006.

3. Manolakos E, Peitsidis P, Eleftheriades M, Dedoulis E, Ziegler M, Orru S, Liehr T and Petersen MB: Prenatal detection of full monosomy 21 in a fetus with increased nuchal translucency: Molecular cytogenetic analysis and review of the literature. J Obstet Gynaecol Res 36: 435-440, 2010.

4. Errichiello E, Novara F, Cremante A, Verri A, Galli J, Fazzi E, Bellotti D, Losa L, Cisternino M and Zuffardi O: Dissection of partial 21q monosomy in different phenotypes: Clinical and molecular characterization of five cases and review of the literature. Mol Cytogenet 9: 21, 2016.

5. Fukai R, Hiraki Y, Nishimura G, Nakashima M, Tsurusaki Y, Saitsu H, Matsumoto N and Miyake N: A de novo 1.4-Mb deletion at 21q22.11 in a boy with developmental delay. Am J Med Genet A 164A: 1021-1028, 2014. 
6. Lyle R, Béna F, Gagos S, Gehrig C, Lopez G, Schinzel A Lespinasse J, Bottani A, Dahoun S, Taine L, et al: Genotype-phenotype correlations in Down syndrome identified by array CGH in 30 cases of partial trisomy and partia monosomy chromosome 21. Eur J Hum Genet 17: 454-466, 2009.

7. Roberson EDO, Wohler ES, Hoover-Fong JE, Lisi E, Stevens EL, Thomas GH, Leonard J, Hamosh A and Pevsner J: Genomic analysis of partial 21q monosomies with variable phenotypes. Eur J Hum Genet 19: 235-238, 2011.

8. Ilbery PL, Lee CW and Winn SM: Incomplete trisomy in a mongoloid child exhibiting minimal stigmata. Med J Aust 48: 182-184, 1961

9. Pelleri MC, Cicchini E, Locatelli C, Vitale L, Caracausi M, Piovesan A, Rocca A, Poletti G, Seri M, Strippoli P, et al: Systematic reanalysis of partial trisomy 21 cases with or without Down syndrome suggests a small region on 21q22.13 as critical to the phenotype. Hum Mol Genet 25: 2525-2538, 2016

10. Wechsler D: Manual for the Wechsler Intelligence Scale for children. 3rd edition. The Psychological Corporation, San Antonio, TX, 1991.

11. American Psychiatric Association: Diagnostic and statistical manual of mental disorders. 5th edition. American Psychiatric Association, Arlington, VA, 2013.

12. ISCN 2016: An International System for Human Cytogenomic Nomenclature. McGowan-Jordan J, Simons A and Schmid M (eds). Cytogenet Genome Res 149: 237-328, 2016.

13. Papoulidis I, Sotiriadis A, Siomou E, Papageorgiou E, Eleftheriades M, Papadopoulos V, Oikonomidou E, Orru S, Manolakos E and Athanasiadis A: Routine use of array comparative genomic hybridization (aCGH) as standard approach for prenatal diagnosis of chromosomal abnormalities. Clinical experience of 1763 prenatal cases. Prenat Diagn 35: 1269-1277, 2015

14. Bonaglia MC, Marelli S, Gottardi G, Zucca C, Pramparo T, Giorda R, Grasso R, Borgatti R and Zuffardi O: Subtelomeric trisomy 21q: A new benign chromosomal variant. Eur J Med Genet 50: 54-59, 2007.

15. Gijsbers ACJ, van Haeringen A, Bosch CAJ, Hansson K, Verschuren M, Bakker E, Breuning MH and Ruivenkamp CAL: A subtle familial translocation $\mathrm{t}(3 ; 21)(\mathrm{p} 26.3 ; \mathrm{q} 22.3)$ : An apparently healthy boy with a $3 p$ deletion and $21 q$ duplication. Cytogenet Genome Res 128: 245-249, 2010.

16. Su MT, Kuan LC, Chou YY, Tan SY, Kuo TC and Kuo PL: Partial trisomy of chromosome 21 without the Down syndrome phenotype. Prenat Diagn 36: 492-495, 2016.

17. Ravnan JB, Tepperberg JH, Papenhausen P, Lamb AN, Hedrick J, Eash D, Ledbetter DH and Martin CL: Subtelomere FISH analysis of 11,688 cases: An evaluation of the frequency and pattern of subtelomere rearrangements in individuals with developmental disabilities. J Med Genet 43: 478-489, 2006.

18. Rowe LR, Lee JY, Rector L, Kaminsky EB, Brothman AR, Martin CL and South ST: U-type exchange is the most frequent mechanism for inverted duplication with terminal deletion rearrangements. J Med Genet 46: 694-702, 2009.

19. Yu S and Graf WD: Telomere capture as a frequent mechanism for stabilization of the terminal chromosomal deletion associated with inverted duplication. Cytogenet Genome Res 129: 265-274, 2010.

20. Falik-Borenstein TC, Pribyl TM, Pulst SM, Van Dyke DL, Weiss L, Chu ML, Kraus J, Marshak D and Korenberg JR: Stable ring chromosome 21: Molecular and clinical definition of the lesion. Am J Med Genet 42: 22-28, 1992.

21. McGinniss MJ, Kazazian HH Jr, Stetten G, Petersen MB, Boman H, Engel E, Greenberg F, Hertz JM, Johnson A, Laca Z, et al: Mechanisms of ring chromosome formation in 11 cases of human ring chromosome 21. Am J Hum Genet 50: 15-28, 1992.

22. Bassett AS, Chow EW and Weksberg R: Chromosomal abnormalities and schizophrenia. Am J Med Genet 97: 45-51, 2000.
23. Janowsky DS and Davis JM: Diagnosis and treatment of depression in patients with mental retardation. Curr Psychiatry Rep 7: 421-428, 2005

24. Strang JF, Kenworthy L, Daniolos P, Case L, Wills MC, Martin A and Wallace GL: Depression and anxiety symptoms in children and adolescents with autism spectrum disorders without intellectual disability. Res Autism Spectr Disord 6: 406-412, 2012.

25. Kaneva RP, Chorbov VM, Milanova VK, Kostov CS, Nickolov KI, Chakarova CF, Stoyanova VS, Nikolova-Hill AN, Krastev SK, Onchev GN, et al: Linkage analysis in bipolar pedigrees adds support for a susceptibility locus on 21q22. Psychiatr Genet 14 : 101-106, 2004.

26. Liu J, Juo SH, Terwilliger JD, Grunn A, Tong X, Brito M, Loth JE, Kanyas K, Lerer B, Endicott J, et al: A follow-up linkage study supports evidence for a bipolar affective disorder locus on chromosome 21q22. Am J Med Genet 105: 189-194, 2001.

27. Poelmans G, Engelen JJM, Van Lent-Albrechts J, Smeets HJ, Schoenmakers E, Franke B, Buitelaar JK, Wuisman-Frerker M, Erens W, Steyaert J, et al: Identification of novel dyslexia candidate genes through the analysis of a chromosomal deletion. Am J Med Genet B Neuropsychiatr Genet 150B: 140-147, 2009.

28. Numata S, Nakataki M, Iga J, Tanahashi T, Nakadoi Y, Ohi K, Hashimoto R, Takeda M, Itakura M, Ueno S, et al: Association study between the pericentrin (PCNT) gene and schizophrenia. Neuromolecular Med 12: 243-247, 2010.

29. Numata S, Iga J, Nakataki M, Tayoshi S, Tanahashi T, Itakura M, Ueno $\mathrm{S}$ and Ohmori T: Positive association of the pericentrin (PCNT) gene with major depressive disorder in the Japanese population. J Psychiatry Neurosci 34: 195-198, 2009.

30. Aleksovska K, Leoncini E, Bonassi S, Cesario A, Boccia S and Frustaci A: Systematic review and meta-analysis of circulating S100B blood levels in schizophrenia. PLoS One 9: e106342, 2014.

31. Dagdan E, Morris DW, Campbell M, Hill M, Rothermundt M, Kästner F, Hohoff C, von Eiff C, Krakowitzky P, Gill M, et al: Functional assessment of a promoter polymorphism in S100B, a putative risk variant for bipolar disorder. Am J Med Genet B Neuropsychiatr Genet 156B: 691-699, 2011

32. da Rosa MI, Simon C, Grande AJ, Barichello T, Oses JP and Quevedo J: Serum S100B in manic bipolar disorder patients: Systematic review and meta-analysis. J Affect Disord 206: 210-215, 2016.

33. Guloksuz SA, Abali O, Aktas Cetin E, Bilgic Gazioglu S, Deniz G, Yildirim A, Kawikova I, Guloksuz S and Leckman JF: Elevated plasma concentrations of S100 calcium-binding protein $\mathrm{B}$ and tumor necrosis factor alpha in children with autism spectrum disorders. Br J Psychiatry 39: 195-200, 2017.

34. Egger G, Roetzer KM, Noor A, Lionel AC, Mahmood H, Schwarzbraun T, Boright O, Mikhailov A, Marshall CR, Windpassinger $\mathrm{C}$, et al: Identification of risk genes for autism spectrum disorder through copy number variation analysis in Austrian families. Neurogenetics 15: 117-127, 2014.

35. Iossifov I, Ronemus M, Levy D, Wang Z, Hakker I, Rosenbaum J, Yamrom B, Lee YH, Narzisi G, Leotta A, et al: De novo gene disruptions in children on the autistic spectrum. Neuron 74: 285-299, 2012

36. Iossifov I, Levy D, Allen J, Ye K, Ronemus M, Lee YH, Yamrom B and Wigler M: Low load for disruptive mutations in autism genes and their biased transmission. Proc Natl Acad Sci USA 112: E5600-E5607, 2015.

37. Kong R, Shao S, Wang J, Zhang X, Guo S, Zou L, Zhong R, Lou J, Zhou J, Zhang J, et al: Genetic variant in DIP2A gene is associated with developmental dyslexia in Chinese population. Am J Med Genet B Neuropsychiatr Genet 171B: 203-208, 2016.

38. Darnell JC, Van Driesche SJ, Zhang C, Hung KY, Mele A, Fraser CE, Stone EF, Chen C, Fak JJ, Chi SW, et al: FMRP stalls ribosomal translocation on mRNAs linked to synaptic function and autism. Cell 146: 247-261, 2011. 